\title{
Painful left shoulder
}

\author{
Jigar N. Patel • Wei-Lien Wang • William A. Murphy Jr.
}

Received: 21 June 2011 /Revised: 28 October 2011 / Accepted: 3 November 2011 /Published online: 1 December 2011

(C) ISS 2011

\section{Diagnosis}

Extranodal primary osseous form of Rosai-Dorfman disease.

\section{Discussion}

Sinus histiocytosis with massive lymphadenopathy (SHML), characterized by histiocyte-laden massive lymph node enlargement, was initially described by Rosai and Dorfman in 1969 as a rare, non-neoplastic and self-limiting disease [1]. The classical presentation of massive symmetrical lymph node enlargement has a predilection for cervical lymph nodes. Extranodal disease, with or without lymphadenopathy, is termed "Rosai-Dorfman disease" (RDD) [2] and most commonly involves the skin, upper respiratory tract, and bone, occurring in approximately $43 \%$ of patients [3]. Osseous lesions are seen in approximately $8 \%$ of patients [3]. Isolated osseous involvement without lymphadenopathy or other extranodal disease as in this case is exceedingly rare [4].

RDD can present at any age but has typically been found in the first or second decade of life, with a mean age of 20.6

The case presentation can be found at doi:10.1007/s00256-011-1325-x.

J. N. Patel $\cdot$ W. A. Murphy Jr. $(\bowtie)$

Diagnostic Radiology,

University of Texas MD Anderson Cancer Center,

1515 Holcombe Blvd. Unit 1475,

Houston, TX 77030-4009, USA

e-mail: wmurphy@mdanderson.org

\section{W.-L. Wang}

Department of Pathology,

University of Texas MD Anderson Cancer Center,

1515 Holcombe Blvd. Unit 085,

Houston, TX 77030-4009, USA years [3]. RDD is generally self-limited with spontaneous resolution; however, it may have a prolonged course with remission, exacerbation, and even death $[3,5]$. After reviewing treatments, Pulsoni et al. suggested observation without treatment is advisable except when compression or other clinical signs indicate surgical debulking [6]. This patient was successfully treated by curettage and bone grafting.

This case demonstrates the typical clinical and osseous imaging characteristics of the uncommon isolated primary osseous form of RDD. The patient had no lymphadenopathy clinically, nor upon CT imaging of the body. Although the patient complained of fatigue and was anemic with a hemoglobin of $8.7 \mathrm{~g} / \mathrm{dl}$, the other often-seen clinical manifestations of fever and weight loss were absent. Other frequently associated lab findings including an elevated erythrocyte sedimentation rate, leukocytosis, reversal of the T4/T8 lymphocyte ratio, and hypergammaglobulinemia [3, 7], were normal or not studied.

The typical radiographic lytic bone lesion of RDD may have a poorly or sharply defined margin, most often in a medullary location. Bone lesions may be single monostotic, multiple monostotic, or polyostotic. As in this case, bone lesions usually do not have periosteal reaction or sclerosis, but these appearances may occur. Intralesional calcification has not been reported. Serial imaging generally reveals a decrease in lesion size leading to complete resolution; sometimes a sclerotic focus remains. $[2,7,8]$

In general, and in this case, the radiographic appearance is that of a semi-aggressive or an aggressive condition that suggests a differential diagnosis including osteomyelitis, multiple myeloma, lymphoma, sarcoma, Langerhans cell histiocytosis, metastatic disease, and rare entities such as lipid storage disorders and sarcoid $[8,9]$. MRI commonly demonstrates elevated signal intensity on T2-weighted sequences, low signal intensity on T1-weighted sequences, soft tissue extension, and adjacent soft tissue edema which 
is likely reactive $[7,8,10]$, but MRI is acknowledged as not very helpful for narrowing the differential diagnosis.

In the present case, the radiographic characteristics indicate an aggressive process with cortical penetration. Because the lesion destroys a segment of cortex, benign conditions that may occur at this age and in this location, such as unicameral bone cysts and fibrous lesions, are not good differential options. Based upon the lack of regional demineralization and periostitis and in the absence of fever and intense pain, osteomyelitis is unlikely. Multiple myeloma and metastases are unlikely due to young age. Lymphoma, sarcoma, Langerhans cell histiocytosis, and sarcoid may occur at this age and may or may not exhibit differentiating features. In this instance, there are no unique radiographic features sufficient to focus the diagnosis on a single entity.

Unfortunately, MRI is of limited value in reduction of the differential to a single diagnosis. In this case, some lesion margins (cancellous and soft tissue) are reasonably well defined, indicative of a less aggressive lesion. Lesion T2 signal intensity is increased but not as great as in most neoplastic conditions. Adjacent bone marrow and soft-tissue edema is prominent about the less well-defined margins of the lesion. Similar features may be encountered in many neoplastic conditions but the mixed pattern of nonaggressive with aggressive borders in the same lesion is somewhat atypical for most aggressive neoplastic lesions.

Because imaging features are somewhat atypical, other less common conditions should be considered. Principal among them is the primary bone form of Rosai-Dorfman disease, a condition of young persons with lower-grade pain and with imaging features of an intermediately aggressive condition. These cases are often diagnostic dilemmas that are only resolved histopathologically.

RDD is classically characterized by histiocytes with abundant eosinophilic to vacuolated foamy cytoplasm and typically with single ungrooved nuclei. Marrow fibrosis and an accompanying mixed inflammatory infiltrate including plasma cells, lymphocytes, neutrophils (sometimes forming microabscesses), and occasional eosinophils are also present. The histiocytes of RDD are CD68 positive, S100 positive, and CD1a negative (a marker for Langerhan cells cells) [4, 7, 11]. They also exhibit the distinctive phenomenon of emperipolesis (a phenomenon also known as lymphophagocytosis) where intact lymphocytes neutrophils, plasma cells, or erythrocytes are phagocytized $[2,10]$. Extranodal disease can sometimes have less lymphophagocytosis and less histiocytes, and therefore be more challenging to diagnose [11].

The present case illustrates the clinical, imaging, and histologic features of RDD in the setting of a solitary osseous lesion. Primary RDD of bone should be considered when an atypical lytic bone lesion is encountered, particularly in a young person.

\section{References}

1. Rosai J, Dorfman RF. Sinus histiocytosis with massive lymphadenopathy: a newly recognized benign clinicopathological entity. Arch Pathol. 1969;87:63-70.

2. Sartoris DJ, Resnick D. Osseous involvement in sinus histiocytosis with massive lymphadenopathy (Rosai-Dorfman disease). Eur J Pediatr. 1986;145:238-40.

3. Foucar E, Rosai J, Dorfman RF. Sinus histiocytosis with massive lymphadenopathy (Rosai-Dorfman disease). Review of the entity. Semin Diagn Pathol. 1990;7:19-73.

4. Demicco EG, Rosenberg AE, Bjornsson J, et al. Primary RosaiDorfman disease of bone - a clinicopathologic study of 15 cases. Am J Surg Path. 2010;34:1324-33.

5. Abdelwahab IF, Klein MJ, Springfield DS, Hermann G. A solitary lesion of talus with mixed sclerotic and lytic changes: RosaiDorfman disease of 25-years' duration. Skeletal Radiol. 2004;33:230-3.

6. Pulsoni A, Anghel G, Falcucci P, Matera R, Pescarmona E, Ribersani M, Villiva N, Mandelli F. Treatment of sinus histiocytosis with massive lymphadenopathy (Rosai-Dorfman disease): report of a case and literature review. Am J Hematol. 2002;69:67-71.

7. Yoon AJ, Parisien M, Feldman F, Young-In Lee F. Extranodal Rosai-Dorfman disease of bone, subcutaneous tissue and paranasal sinus mucosa with a review of its pathogenesis. Skeletal Radiol. 2005;34:653-7.

8. Lin J, Lazarus M, Wilbur A. Sinus histiocytosis with massive lymphadenopathy: MRI findings of osseous lesions. Skeletal Radiol. 1996;25:279-82.

9. George J, Stacy G, Peabody T, Montag A. Rosai-Dorfman disease manifesting as a solitary lesion of the radius in a 41 -year-old woman. Skeletal Radiol. 2003;32:236-9.

10. Sundaram C, Shantveer U, Chandrashekar P, Prasad VBN, Umadevi M. Multifocal osseous involvement as the sole manifestation of Rosai-Dorfman disease. Skeletal Radiol. 2005;34(10):658-64.

11. Dorfman H, Czerniak B. Bone tumors. 1st ed. St. Louis, Missouri: Mosby; 1998. p. 704-15. 